\title{
Effectiveness, Moderators and Mediators of Self-regulation Intervention on Older Adults' Exercise Behavior: a Randomized, Controlled Crossover Trial
}

\author{
Kazuhiro Harada ${ }^{1}$ (D) \\ Accepted: 11 December 2021 / Published online: 7 January 2022 \\ (c) International Society of Behavioral Medicine 2022
}

\begin{abstract}
Background Although self-regulation interventions are effective in promoting exercise behaviors, moderators and mediators of interventions among older adults are not well established. This study aimed to examine whether (1) self-regulation intervention promoted exercise behavior, (2) health literacy and habit strength moderated the intervention effect, and (3) self-regulation and habit strength mediated the intervention effect among older adults.

Methods This study was a randomized, non-blinded, controlled crossover trial. The baseline questionnaire survey assessed the average amount of exercise time per day, self-regulation, habit strength, health literacy, and socio-demographic factors. After the baseline survey, 393 community-dwelling older adults were randomly assigned to either the immediate intervention or the delayed intervention group. For the immediate group, print-based materials were provided once a week for 7 weeks before a second questionnaire survey. For the delayed group, the materials were provided only after the second survey. Finally, a third survey was conducted for both groups.

Results The mixed models showed that the average exercise time was increased after the intervention in both groups. Multiple regression analyses revealed that no factor moderated the intervention effect. From the path analyses, the mediating effect of self-regulation on the relationship between intervention and changes in average exercise time was supported, but the mediating role of habit strength was not clearly indicated.

Conclusions Although the mediating roles of habit strength for the intervention effects are still inconclusive, self-regulation intervention can promote exercise behavior among older adults, regardless of their health literacy level, habit strength, and socio-demographic characteristics.
\end{abstract}

Keywords Exercise $\cdot$ Habits $\cdot$ Health literacy $\cdot$ Healthy aging $\cdot$ Self-control

\section{Introduction}

The health benefits of physical activity among older adults are well established. Physical activities are defined as all activities that require energy expenditure $[1,2]$. Current physical activity guidelines set by the World Health Organization (WHO) recommend that older adults should engage in at least 150-300 min per week of moderate-intensity aerobic physical activity, 75-150 min per week of vigorous-intensity

Kazuhiro Harada

harada@harbor.kobe-u.ac.jp

1 Active Aging Research Hub, Graduate School of Human Development and Environment, Kobe University, 3-11

Tsurukabuto, Nada, Kobe 657-8501, Japan physical activity, or an equivalent combination of the two, for healthy living [1]. In Japan, the Ministry of Health, Labour, and Welfare recommends that Japanese older adults engage in physical activity at least $40 \mathrm{~min}$ per day regardless of level of intensity [2]. However, the level of physical activity among older adults is lower than among younger adults [3]. The development and dissemination of effective strategies to increase physical activity among older adults is a public health priority. Physical activity occurs in various domains, such as leisure time, occupation, household, and transportation [1]. After retirement, older adults are likely to lose the opportunity to engage in physical activities during occupations and transportation [4]. Increasing physical activity during leisure time would be especially important to compensate for the decrease in opportunities for physical activity during occupation and transportation among those 
who are withdrawing from social roles through retirement. Exercise is a major component of physical activities that occur during leisure time [1]. Exercise is defined as physical activity that is planned, structured, repetitive, and designed to promote physical fitness and health [1,2]. The Ministry of Health, Labour, and Welfare recommends that Japanese citizens of all ages engage in exercise at any level of intensity at least 30 min per day, twice a week or more [2].

Self-regulation is a widely accepted strategy for promoting physical activity. Self-regulation is an umbrella term that describes the pursuit and attainment of goals [5] and generally refers to an individual's efforts to modify thoughts, feelings, desires, and actions to attain higher goals [6]. The concept of self-regulation is incorporated into health behavioral theories/models [7], such as the Temporal Self-regulation Theory [8] and Social Cognitive Theory [9]. Concepts that largely overlap with self-regulation are also included in various theories and models (e.g., process of change in the Transtheoretical Model [10]; action planning and coping planning in the Health Action Process Approach [11]). Health behavior interventions have commonly employed self-regulation strategies to promote health behaviors [12]. Major self-regulation strategies included in physical activity interventions are goal setting, review of behavior goals, self-monitoring of behavior, action planning (including implementation intention), problem-solving (including relapse prevention and coping planning), reducing negative emotions, self-talk, time management, and feedback on behavior [13]. For physical activity, interventional [14] and observational studies [15] have consistently indicated that self-regulation is an important factor in explaining and promoting physical activity among adults in the general population. Review articles [16] for the mediators of intervention have also proposed the importance of self-regulation in physical activity behavior change.

The effectiveness of self-regulation strategies to promote physical activity among older adults has been also reported. Although a meta-analysis published in 2014 [17] concluded that common self-regulation techniques for modifying physical activity in younger adults would not be effective for older individuals, recent systematic reviews and meta-analyses support the effectiveness of such interventions [18-21]. One meta-analysis of cardiovascular disease studies that included older patients reported that interventions employing self-monitoring can increase levels of physical activity [18]. A meta-analysis of patients with non-communicable diseases, including older patients [19], showed that multiple health behavior change interventions based on the information technology can promote physical activity, and common interventions have employed self-regulation strategies. In non-clinical settings, reviews $[20,21]$ showed that interventions using information technology can increase physical activity among older adults, and majority of such interventions have employed goal setting. Evidence for the effectiveness of self-regulation interventions among older Japanese individuals, however, is still limited.

To understand the effectiveness of self-regulation interventions among older adults more extensively, identifying moderators (effect modifiers) of the intervention is helpful. Identification of moderating factors can predict who is likely to succeed or fail to implement behavioral change through such interventions [22, 23]. Health literacy may be a moderator of self-regulation interventions. People's health literacy tends to decline as they get older [24, 25]. A systematic review showed that lower health literacy was associated with lower physical activity levels [26]. A conceptual model proposes that health literacy impacts health conditions through increasing health behaviors, including physical activity [27, 28]. As health literacy is generally defined as the ability to access, understand, appraise, and apply health-related information [29], it can be assumed that those with higher health literacy could understand, appraise, and apply self-regulation strategies more easily, thereby succeed in behavior change more definitively than those with lower health literacy. However, as the systematic review indicated [26], the moderating role of health literacy in the intervention effects on physical activity behavior change is still overlooked. Only a few studies have examined the moderating role of health literacy in physical activity interventions, among younger to middle-aged populations [30, 31]. It remains unclear whether health literacy moderates the effectiveness of self-regulation interventions for physical activity among older adults.

Along with health literacy, habit strength may also moderate self-regulation interventions. Habit strength is recognized as a core research topic in the area of physical activity behavior change [32]. Habit strength is conceptualized as specific actions or tendencies occurring with little consciousness or reflection in response to specific contextual cues [32]. When a habit is adequately formed, contextual cues are assumed to promote behavior automatically, without deliberate efforts or conscious motivations [33]. This assumption is consistent with the impulsive process in dual-process models [34]. Previous studies of smoking behavior [35] in high school students and physical activity of university students [36] reported that planning interventions, a major selfregulation strategy, were more effective in individuals with lower habit strength than in those with higher habit strength. Results, viewed conceptually, suggest that the pre-existing habit was more likely to interfere with the learning of new associations between the contextual cues and behavior in those with higher habit strength $[35,36]$. These studies among students $[35,36]$ suggest that selfregulation interventions have a greater impact on those with lower habit strength compared to those with higher habit strength. Another study using a sample of adults, 
however, reported a null result for the moderating role of habit strength in the effect of planning intervention on physical activity behavior change [37]. More extensive research among various populations is needed to evaluate the moderating role of habit strength in self-regulation interventions.

Furthermore, besides being moderators, clarifying the mediating process of interventions on behavior change is also helpful to better understand the effectiveness of selfregulation interventions among older adults. Clarifying the mediating process can guide our understanding of mechanisms for intervention effects, strengthen the theoretical basis of the link between intervention and behavior change, and suggest further improvements in intervention strategies $[23,38]$. According to the framework for understanding habit formation and its determinants [33, 39, 40], as well as moderating role, habit strength might play a mediating role in the effect of self-regulation interventions on physical activity: increased self-regulation by the intervention reinforces the habit, and in turn, the reinforced habit induces desirable behavior change. It is proposed that continuous employment of self-regulation could gradually form a habit by translating the reflective process into an impulsive process $[33,39,40]$. A review of self-regulation also argued that attempting to automate behavior is important for successful self-regulation [5]. Intervention studies have employed planning to form habits [41]. However, although self-regulation strategies are widely accepted as a way of forming a habit, few intervention studies have confirmed their mediation process. Examining the mediation process of self-regulation interventions could advance current knowledge about habit formation.

Focusing on exercise behavior from the various aspects of physical activity, the present study examined (1) whether the self-regulation intervention promoted exercise behavior, (2) whether health literacy and habit strength moderated the intervention effect, and (3) whether self-regulation and habit strength mediated the intervention effect among older adults. In particular, the present study hypothesized that exercise behavior will increase after the self-regulation intervention (main effect, Hypothesis 1); the increase of exercise behavior accompanied by the intervention will be larger among those with higher health literacy than those with lower health literacy (moderating effect, Hypothesis 2-1), and among those with lower habit strength than those with higher habit strength at baseline (moderating effect, Hypothesis 2-2). The increase of exercise behavior measured from pre- to post-intervention will be mediated by changes in self-regulation and habit strength during the same period (mediating effect, Hypothesis 3 ).

\section{Methods}

\section{Study Design}

This study was a non-blinded, randomized controlled crossover trial. This study followed the CONSORT 2010 statement: extension to randomized crossover trials (Electronic Supplementary Material 1) [42]. The crossover design is feasible and equitable in community-based settings. Figure 1 shows the flow diagram of the intervention. Treating exercise behavior as the main outcome, the present study distributed the questionnaire surveys three times to the participants by postal mail. The baseline survey was conducted until October 22, 2020; after that, the participants were randomly allocated to the immediate or delayed intervention groups in a 1:1 ratio, using computer-generated random numbers. The randomization was conducted simultaneously by an assistant staff member who did not know the research purpose. To avoid contamination of the intervention within married couples due to the mailing of print-based materials, the randomization was stratified by those who enrolled accompanying spouses and those who enrolled alone, and married couples were assigned to the same group. The group allocation was not blinded.

The print-based intervention materials were mailed to the immediate intervention group for every Friday for 7 weeks from October 30 to December 11, 2020. The second survey was conducted from December 15 to 26, 2020. The same intervention materials were provided to the delayed intervention group for 7 weeks from January 8 to February 19, 2021. Finally, the third survey was conducted from February 24 to March 7, 2021.

Written informed consent was obtained from all participants. The present study received prior approval (no. 443) from the Ethical Committee of the Graduate School of Human Development and Environment, Kobe University. All procedures were performed in accordance with the Declaration of Helsinki. Prior to participant recruitment, the trial protocol (ID: UMIN000041990) was preregistered at the University Hospital Medical Information Network clinical trials registry, an authorized clinical trial registry of the International Committee of Medical Journal Editors. The protocol was not changed after the registration.

\section{Participants}

The present study calculated the required sample size using $G^{*}$ Power 3.1 for within-between interaction of group and time in a repeated-measurement analysis of 


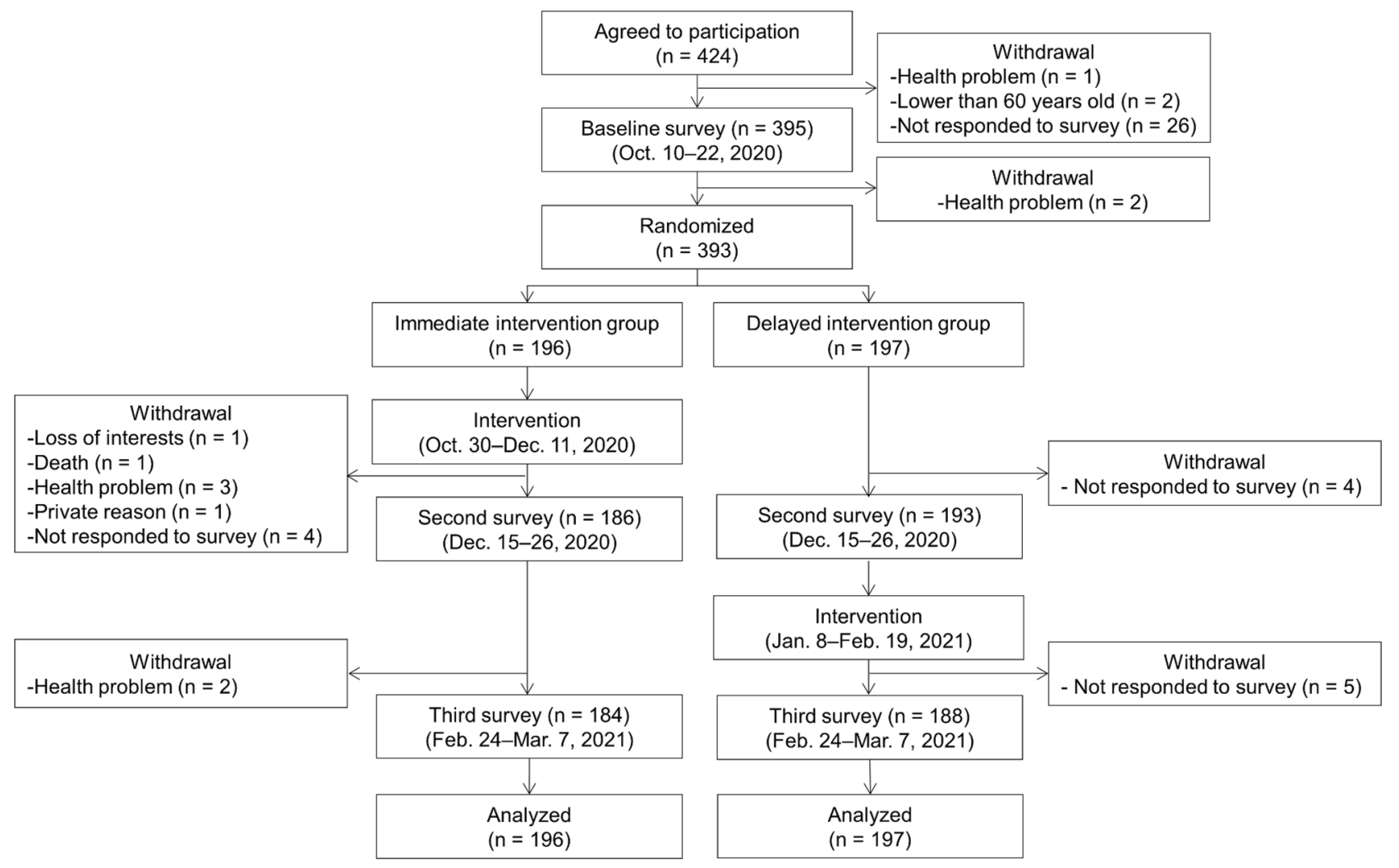

Fig. 1 Flow of the participants through the study

variance [43] prior to recruitment. The intervention effect size on the main outcome was input as small $(f=0.10)$, the alpha error as 0.05 , the power as 0.95 , and the correlation among repeated measures as 0.50 . As the present study did not conduct prior pilot trials, a conservative estimate was judged to be appropriate. The small effect size and higher power were derived from this decision. The analysis indicated that a sample size of 260 was required. To allow $15 \%$ for dropouts, a sample of approximately 300 participants was collected. The $15 \%$ dropout rate was estimated from recent Japanese community-based health behavior intervention studies, which have reported dropout rates of $2.2 \%$ [44], 5.9\% [45], 13.0\% [46], and $13.6 \%$ [47], respectively. The present study employed a more conservative dropout rate than any of these studies [44-47]. If the present study had not reached this sample size at the planned closing date of the recruitment, the present study would have expanded the closing date and distributed the flayers once again using inserts into newspapers. Because the present study recruited the participants simultaneously, the present study did not terminate the enrollment of the participants beyond the sample size of approximately 300 .

Participants were recruited through flyers inserted into the newspaper for all readers living in Nada ward, Kobe City,
Japan, on September 29 and October 1, 2020. The present study also asked the health and healthcare division in the Nada ward office to distribute flyers through their related organizations. Kobe City is one of the major urban cities in Japan and consists of 9 wards. There are approximately 1,515,000 and 136,000 residents in Kobe City and Nada ward, respectively.

The eligibility criteria for participation were as follows: individuals (1) were aged 60 years or above, and (2) had no restrictions on exercise participation. Due to a community-based practical trial, any other inclusions/exclusions were not considered for the present study, and the restrictions of it were just based on self-report. The closing date of the trial recruitment was planned as October 10, 2020.

Among the 424 individuals who agreed to participate in the trial, the 393 individuals who met the eligibility criteria and responded to the baseline survey were randomly assigned to the immediate or delayed intervention groups. The sample included 60 married couples (120 individuals). Three hundred seventy-two individuals $(94.7 \%)$ completed the third survey. No important harm or unintended effects were reported by the participants in either group during the intervention period. No financial incentives were provided to participants. 


\section{Interventions}

The present study delivered print materials once a week for seven consecutive weeks by postal mail. Among the various delivery modes of the interventions, the present study employed print delivery, because the pandemic of COVID19 made it difficult to conduct face-to-face interventions, and a considerable number of older adults are not familiar with the Internet in Japan.

As shown in Electronic Supplementary Material 2, the print material for each week consisted of three components with eight pages of A4 paper, in color: (1) information about exercise and health promotion, (2) information about tips for effective techniques for changing one's behavior, and (3) a form to be filled out for the self-regulatory strategy practices. At the final week, leaflets on health promotion policies and practices in the local community, which were offered by the staff of the health and healthcare division in the Nada ward office, were also delivered to the participants.

The first component each week corresponded with information about health consequences (No. 5.1) in the Behavioral Change Technique Taxonomy version 1 (BCTTv1) [48]. The present study aimed to attract the participants providing the latest evidence for exercise and health promotion.

The second component featured various types of information shared weekly with participants. Tips for effective self-monitoring shared in the first week aligned with self-monitoring of behavior (No. 2.3 in BCTTv1). Tips for effective goal setting and action planning given in the second week corresponded to goal setting (behavior) (No. 1.1 in BCTTv1) and action planning (No. 1.4 in BCTTv1). In the third week, tips for creating and keeping desirable motivation levels aligned with pros and cons (No. 9.2 in BCTTv1). Tips for receiving effective social support in the fourth week corresponded to social support (practical) (No. 3.2 in BCTTv1) and social support (emotional) (No. 3.3 in BCTTv1). Tips for preventing relapse and coping barriers during the fifth week aligned with problem solving (No. 1.2 in BCTTv1). The sixth week's tips for building selfconfidence to maintain exercise behavior corresponded to review behavior goal(s) (No. 1.5 in BCTTv1) and focus on past success (No. 15.3 in BCTTv1). The third component was supplemented by building on information from the second component.

The third component was designed as the main part of the intervention. It provided three types of fill-out forms. Three fill-out forms corresponded to (1) goal setting (behavior) (No. 1.1 in BCTTv1) and action planning (No. 1.4 in BCTTv1); (2) self-monitoring of behavior (No. 2.3 in BCTTv1); and (3) review behavior goal $(s)$ (No. 1.4 in BCTTv1), respectively. The present study focused on these self-regulation techniques because it would be sufficiently feasible and effective to provide information about them via weekly distribution of printed materials during the COVID19 pandemic. On the fill-out form for goal setting (behavior) and action planning, at the beginning of the week, the study recommended that the participants plan and fill out (1) how many days they would exercise during that week, (2) the timing or context for doing exercise on the designated days, and (3) total duration of exercise on each of the days. For the fill-out from related to self-monitoring of behavior, participants were advised to daily check and fill out (1) whether they originally planned that day for exercise, (2) whether they actually did exercise on that day, (3) total duration time of exercise, and (4) total step counts every day regardless of whether it was a designated exercise day. On the fill-out form for review behavior goal (s), at week's end, they were encouraged to review their daily monitoring records and complete a self-reflection about the overall achievements of their daily plans for the week.

\section{Measures}

\section{Exercise Behavior (Main Outcome)}

Exercise behavior was the main outcome of this study as planned. The study asked participants to answer the number of days they engaged in exercise in a usual week ( 0 to 7 days) in every survey. If they answered 1 to 7 days, the present study also asked them to indicate the average exercise time (hours and minutes) for days when they engaged in exercise. Walking for exercise, calisthenics, and sports were listed as examples. The weekly exercise time (hours per week) was calculated by multiplying the frequency by time.

Japanese studies [49-51], including the Japan National Health and Nutrition Survey conducted by the Ministry of Health, Labour, and Welfare [52], have simply measured frequencies and durations of exercise in a typical week. Such studies have included all intensities of exercise behavior and have not limited the intensities to the moderate-to-vigorous range. The present study followed these Japanese studies [49-52].

\section{Self-Regulation of Exercise}

The scale developed by Takeda et al. [53] was employed to measure the self-regulation of exercise. It was developed to measure major self-regulatory behavior change techniques for exercise. Taketa et al. [53] regarded goal-setting, selfmonitoring, gathering information, stimulus control, and self-reinforcement as major techniques. Thus, the scale was designed to assess them. The participants were asked to answer this scale in every survey. This scale consists of five self-regulation items. The instruction given was "how often did you do the following during the last 1 month?", and the item examples were "I set realistic goals to do exercise" 
(goal setting) and "I kept the records about my exercise" (self-monitoring) [53]. This scale employs a five-point Likert scale to answer these items. The range is "never" (1), "rarely" (2), "neither" (3), "sometimes" (4), and "often" (5) [53]. Answers were summed across the five items, with higher scores representing higher self-regulation (range: $5-25)$. The construct validity of this scale $(\mathrm{GFI}=0.98$, $\mathrm{AGFI}=0.95, \mathrm{CFI}=0.97, \mathrm{RMSEA}=0.08)$ and internal consistency (Cronbach's $\alpha=0.78$ ) were confirmed by Takeda et al. [53].

\section{Habit Strength of Exercise}

The present study used the Self-Report Behavioral Automaticity Index [54], which is a subscale of the Self-Report Habit Index [55] and is commonly used in psychological research on habits [56], to assess the habit strength of exercise at every surveys. Internal inconsistencies (Cronbach's alpha) of this index for exercise behavior in the previous studies were ranged from 0.70 to 0.96 [54]. Due to lack of a suitable Japanese version, the index was translated into Japanese. Following the instruction, "Doing exercise is something...," the subscale of this index contains four items: "I do automatically," "I do without having to consciously remember," "I do without thinking," and "I start doing before I realize I'm doing it" [54]. A seven-point Likert scale was employed from " 1 " (strongly agree) to "7" (strongly agree), and its score was calculated by summing up the responses of these four items (range: 7-28).

\section{Health Literacy}

At the baseline survey, the existing health literacy scale [57] was used to assess the health literacy level. This scale includes five items. Each item is rated on a five-point scale. Internal inconsistency (Cronbach's alpha) of the scale was 0.86 [57]. Construct validity of the scale was indicated by examining associations with health behaviors, coping styles, and somatic symptoms [57]. Following Ishikawa et al. [57], the average scores for the five items were calculated (range: $1-5)$.

\section{Socio-Demographic Factors}

Sex (men, women), age, educational background ( $<4$-year college, $\geq 4$-year college), current marital status (single, married), living arrangement (alone, with others), perceived economic status (single five-point Likert scale from very poor [1] to very good [5]), and frailty score at baseline were included as socio-demographic factors. Frailty scores were measured using the Kihon checklist [58, 59]. This checklist comprises 25 items, each of which is answered "yes" or "no" and the answers to all items were then summed (range: 0 to
25). Satake et al. [60] showed that Spearman's correlation coefficient between the score of the Kihon checklist and the number of frailty phenotypes was 0.66 .

\section{Perceived Adherence and Acceptance of Intervention}

The present study investigated perceived adherence and acceptance of the intervention in the second survey for the participants in the immediate intervention groups and at the third survey for, all groups. The items are listed in Electronic Supplementary Material 4 . The participants asked to answer these five items from " 0 " (not at all) to "10" (at all).

\section{Analyses}

\section{Psychometric Analyses of Habit Strength}

Using AMOS version 25.0 (IBM Japan, Ltd., Tokyo, Japan), confirmatory factor analysis with a one-factor structure was performed on baseline data for habit strength. Chi-square, comparative fit index (CFI), Tucker-Lewis index (TLI), and root-mean-square error of approximation (RMSEA) were examined as model fit indices [61]. The cut-off for CFI and TLI was 0.95 and 0.06 for RMSEA [61]. If the model fit indices in the initial model did not reach the cut-off values, it was revised by adding one correlated error.

Analyzing the baseline data using SPSS for Windows v.25.0 (IBM Japan, Ltd., Tokyo, Japan), Cronbach's alpha and Pearson's $r$ coefficient for correlation with average exercise time were calculated. Using baseline and second survey data among the delayed intervention group, Pearson's correlation coefficient for test-retest was also calculated.

\section{Main Effect of Intervention on Exercise Behavior}

The present study used linear mixed models to investigate the main effects of intervention on exercise behavior. Linear mixed models were constructed using the mixed command of Stata v.14 (StataCorp LLC, College Station, TX, USA). Two models were examined by setting the average exercise time as the dependent variable. In model 1, group $(0=$ delayed group, $1=$ immediate group $)$, survey point (baseline survey $=0$, second survey $=1$, third survey $=2$ ), and the interaction terms of the group with the survey point were examined as the independent variables. In model 2 , in addition to the variables in model 1 , socio-demographic factors at baseline (age, sex $[\operatorname{men}=0$, women $=1]$, age, educational background $[<4$-year college $=0, \geq 4$-year college $=1$ ], marital status [ single $=0$, married $=1$ ], living arrangement [alone $=0$, others $=1$ ], perceived economic status, and frailty score) and enrollment with spouse (no $=0$, yes $=1$ ) were also included as independent variables. These independent variables were treated as fixed effects, and 
unstandardized and standardized regression coefficients were calculated. The variance of intercept for individuals was treated as a random effect. The random effect was estimated based on the variance components' covariance structure. If the interactive effect of the immediate group with the second survey was statistically significant, the interactive effect of the immediate group with the third survey was not significant, and the main effect of the third survey was significant; this would be interpreted as supporting Hypothesis 1 . The mixed models estimated the difference and $95 \%$ confidence interval of the average exercise time across each survey point within each group. The confidence intervals and $p$-values were corrected using Bonferroni's method. As 15 comparisons were examined in the mixed command of Stata v.14 for the interaction of the group with the survey point in the case of the present study, $0.00333(=0.05 / 15)$ was set as the statistical significance level for Bonferroni's method. If exercise time increased from baseline to the second survey among the immediate group and from the second to the third survey among the delayed group, these results would support Hypothesis 1.

Since mix models can estimate missing values, the present study included all participants. Thus, an intention-totreat analyses was done for the main effect of the intervention. The maximum likelihood estimation was used to fit the model. Significance was set at $p<0.05$.

\section{Moderators of Intervention Effects on Exercise Behavior}

The present study conducted multiple regression analyses to investigate the moderators of the intervention effect on exercise behavior separately for two study phases (baseline to second survey; second to third survey). By examining concordances of the results between the two study phases, the present study can strengthen the reproducibility of the findings. To assess the moderating role of health literacy and habit strength, regression analyses were performed using two models. The dependent variables were average exercise time at the second survey for the previous phase and the average exercise time at the third survey for the latter phase. In both models, the last observation values of the average exercise time (the average exercise time at the baseline survey for the previous phase; the average exercise time at the second survey for the latter phase), the group, health literacy at baseline, habit strength at baseline, the group's interaction term with health literacy, the group's interaction term with habit strength, the socio-demographic factors and enrollment with spouse were included as independent variables by the forced-entry method. Model 1 included the participants without missing data (complete case analysis). Imputing missing data by the multiple imputation method with the Markov chain Monte Carlo approach (30 datasets), model 2 included all participants. As well as unstandardized regression coefficients in both models, the standardized regression coefficients in model 1 were also estimated to indicate the effect sizes of the intervention and the moderations. If the group's interaction terms with health literacy regressed significantly and positively on the dependent variable during the previous phase and regressed negatively at the latter phase, these results would support Hypothesis 2-1. If the group's interaction term with habit strength regressed significantly and negatively at the previous phase and positively at the latter phase, the results would support Hypothesis 2-2. If regressions of any interaction terms were significant, the stratified analyses by mean and standard deviation (SD) were conducted.

This study also conducted additional regression analyses to explore whether any socio-demographic factors and enrollment with spouse moderated the intervention effects. In total, eight interaction terms with socio-demographic factors along with enrollment with spouse were calculated for the group. Then, the eight interaction terms were added in model 1 by the stepwise method. If any interaction term(s) were selected in the model, stratified analyses were conducted.

Prior to calculating the interaction term and conducting the analyses, continuous variables were mean-centered. Significance was set at $p<0.05$. Moderation analyses were performed using SPSS for Windows v.25.0 (IBM Japan, Ltd., Tokyo, Japan).

\section{Mediation Process of Intervention Effects on Exercise Behavior}

The total effects of the intervention on self-regulation and habit strength were examined using linear mixed models. The procedures of the mixed models for self-regulation and habit strength were the same as the mixed models for the main effects on average exercise time. The independent variables of model 1 were group, survey point, and interaction terms of the group with the survey point. Model 2 added the socio-demographic factors and enrollment with spouse in model 1.

Then, the study conducted path analyses to investigate the mediation process of the intervention effects on exercise behavior separately for two study phases (baseline to second survey; second to third survey). Significance was set at $p<0.05$. Missing values were treated using pairwise deletion. As with previous studies [62, 63], this study calculated and analyzed residualized change scores of average exercise time, self-regulation, and habit strength for each phase. The main analyses examined the sequential mediation model. This model specified six main paths: (a) path from the group to changes in self-regulation, (b) path from the group to changes in habit strength, (c) path from the group to changes in average exercise time, (d) path from changes 
in self-regulation to changes in habit strength, (e) path from changes in self-regulation to changes in average exercise time, and (f) path from changes in habit strength to changes in average exercise time. In addition to these main paths, the additional paths from these socio-demographic factors and/ or from enrollment with spouse to changes in self-regulation, habit strength, and average exercise time were included in the model if statistically significant Pearson correlations of their relationships were observed. Chi-square, CFI, TLI, and RMSEA were evaluated as model fit indices [61]. Among the main paths in the sequential mediation model, if paths (a), (d), and (f) were statistically significant and the path (c) was statistically non-significant, the result would support Hypothesis 3.

The bias-corrected bootstrap method (5000 bootstrap samples) was used to estimate direct, indirect, and total effects, and 95\% confidence intervals of the group, changes in self-regulation, and changes in habit strength on changes in average exercise time in the sequential mediation model. The standardized direct, indirect, and total effects and $95 \%$ confidence intervals were also estimated to indicate the effect sizes of the intervention and the mediations. If the indirect and total effects of the group, the total effect of changes in self-regulation, and the direct and total effect of changes in habit strength were statistically significant, and the direct effects of the group were statistically nonsignificant, the results would support Hypothesis 3 .

As an additional analysis, the present study also examined the parallel mediation model. As this model assumes that the mediation roles of self-regulation and habit strength were parallel, it did not specify the path from changes in self-regulation to those occurring in habit strength. Other paths in the parallel model were the same as paths in the sequential mediation model. By comparing the model fit indices between the sequential and parallel models, the study investigated which mediation model better explains the data.

The path analyses were conducted using AMOS v.25.0 (IBM Japan, Ltd., Tokyo, Japan).

\section{Results}

\section{Baseline Characteristics of Participants}

Table 1 shows the characteristics of the participants at baseline. There were 165 men and 228 women. The mean age was 74.0 years $(\mathrm{SD}=6.5$ years). On average, they engaged in exercise for $37.0 \mathrm{~min}(\mathrm{SD}=40.7 \mathrm{~min})$ per day. Chi-squared tests (for categorical variables) and $t$-tests (for continuous variables) revealed that there were no significant differences in baseline characteristics between the intermediate and delayed intervention groups.

\section{Psychometric Characteristics of Habit Strength}

The means and SD of each item and results of confirmatory factor analysis are reported in Electronic Supplementary Material 3. Although the initial model without correlated error term did not show adequate model fit indices $\left(\chi^{2}[2]=60.7(p<0.001), \mathrm{CFI}=0.949\right.$, TLI $=0.847$, RMSEA $=0.275$, the model fit indices of revised model containing the correlated error between item 1 and item 2 met their cut-off $\left(\chi^{2}[1]=1.0[p=0.307]\right.$, CFI $>0.990$, TFI $>0.999$, RMSEA $=0.011)$.

Table 1 Baseline characteristics of participants and comparison between immediate and delayed intervention group

\begin{tabular}{|c|c|c|c|c|c|}
\hline & $n$ & Total & $\begin{array}{l}\text { Delayed intervention } \\
\text { group }(n=197)\end{array}$ & $\begin{array}{l}\text { Immediate intervention } \\
\text { group }(n=196)\end{array}$ & $p$-value \\
\hline Age (years), M (SD) & 393 & $74.0(6.5)$ & $74.0(6.3)$ & $73.9(6.7)$ & $0.938^{\mathrm{a}}$ \\
\hline Sex (women), $\%$ & 393 & $58.0 \%$ & $57.4 \%$ & $58.7 \%$ & $0.792^{\mathrm{b}}$ \\
\hline Educational background (4-year college) & 387 & $48.1 \%$ & $47.2 \%$ & $49.0 \%$ & $0.720^{\mathrm{b}}$ \\
\hline Marital status (married), $\%$ & 391 & $72.6 \%$ & $73.5 \%$ & $71.8 \%$ & $0.710^{\mathrm{b}}$ \\
\hline Living arrangement (with others), $\%$ & 390 & $82.3 \%$ & $80.5 \%$ & $84.10 \%$ & $0.353^{\mathrm{b}}$ \\
\hline Perceived economic status (score, $1-5$ ), M (SD) & 391 & $3.3(0.7)$ & $3.3(0.7)$ & $3.3(0.7)$ & $0.736^{\mathrm{a}}$ \\
\hline Frailty (score, 0-25), M (SD) & 381 & $4.8(3.1)$ & $4.5(3.0)$ & $5.0(3.1)$ & $0.115^{\mathrm{a}}$ \\
\hline Enrollment with spouse, $\%$ & 393 & $30.5 \%$ & $30.5 \%$ & $30.6 \%$ & $0.973^{\mathrm{b}}$ \\
\hline Health literacy (score, 1-5), M (SD) & 389 & $3.9(0.6)$ & $3.9(0.6)$ & $3.9(0.6)$ & $0.694^{\mathrm{a}}$ \\
\hline Average exercise time (minutes per day), M (SD) & 374 & $37.0(40.7)$ & $40.3(46.5)$ & $33.6(33.4)$ & $0.112^{\mathrm{a}}$ \\
\hline Self-regulation of exercise (score, 5-25), M (SD) & 390 & $12.6(4.7)$ & $13.0(4.8)$ & $12.2(4.5)$ & $0.099^{\mathrm{a}}$ \\
\hline Habit strength of exercise (score, 4-28), M (SD) & 390 & $16.0(6.2)$ & $16.6(6.0)$ & $15.5(6.3)$ & $0.078^{\mathrm{a}}$ \\
\hline
\end{tabular}

${ }^{\mathrm{a}} t$-test, ${ }^{\mathrm{b}}$ chi-squared test

$M$, mean; $S D$, standard deviation

The sample size of each variable was different due to missing values 
The Cronbach's alpha was 0.90 . The Pearson's correlation between habit strength and average exercise time at baseline was 0.44 . The test-retest correlation of the delayed intervention group was 0.66 .

\section{Perceived Adherence and Acceptance of Intervention}

Descriptive statistics of the scores of the items for perceived adherence and acceptance of the intervention are shown in Electronic Supplementary Material 4. T-tests indicated that there were no significant differences in the scores between the immediate and delayed intervention groups.

\section{Main Effects of Intervention on Exercise Behavior}

Electronic Supplementary Material 5 shows the fixed effects in the mixed models for the effects of intervention on exercise behavior. For Hypothesis 1, significant interactive effects of the immediate group with the second survey, nonsignificant interactive effects of the immediate group with the third survey, and the significant main effects of the third survey were detected. These results supported Hypothesis 1 .

Table 2 represents the estimated differences in average exercise time from the baseline to the second and third surveys within each group. Figure 2(a) plots the estimated average exercise time at each survey point. As shown in Table 1 and Fig. 2(a), the intermediate intervention group significantly increased their average exercise time from the baseline to the second survey, and maintained their average exercise time from the second to the third survey. Among the delayed intervention group, while the average exercise time did not significantly change from the baseline to the second survey, it significantly increased from the second to the third survey. These results supported Hypothesis 1.

\section{Moderators of Intervention Effects on Exercise Behavior}

Table 3 presents the results of examining the moderating roles of health literacy and habit strength in the intervention effects. In both models (models 1 and 2) and both study phases (baseline to second survey; second to third survey), the main effects of the group significantly regressed on changes in average exercise time. However, the interaction terms of the group with health literacy and habit strength did not significantly regress on changes in it. These results did not support Hypotheses 2-1 and 2-2.

In the additional regression analyses, any interaction terms with the socio-demographic factors and enrollment with spouse for the group were not selected in the model by the stepwise method.

\section{Mediation Process of Intervention Effects on Exercise Behavior}

The fixed effects in the mixed models for self-regulation and habit strength are shown in Electronic Supplementary Material 6 and Electronic Supplementary Material 7. Table 2 represents estimated differences of self-regulation and habit strength among each survey point within each group. Figure 2(b) and (c) plot the estimated scores at each survey point. As shown in Table 2 and Fig. 2(b) and (c), the intermediate intervention group significantly elevated their scores of self-regulation and habit strength from baseline to the second survey, and kept the score of habit strength at the third survey. Though the score of self-regulation was significantly decreased from the second to third survey, the score of it at the third survey was still significantly higher than the baseline survey among the intermediate intervention group. Among the delayed intervention group, while self-regulation and habit strength did not significantly change from the baseline to the second survey, they were significantly increased from the second to the third survey.

Electronic Supplementary Material 8 shows Pearson's correlations of socio-demographic factors with changes in average exercise time, self-regulation, and habit strength. Frailty significantly correlated with changes in habit strength from the baseline to the second survey, changes in self-regulation from the second to the third survey, and changes in habit strength from the second to the third survey. Age was significantly correlated with changes in habit strength from the second to the third survey.

Figure 3 represents the path models for the sequential mediation model of the intervention on exercise behavior. At both study phases, the group significantly regressed on changes in self-regulation, and changes in self-regulation significantly regressed on both changes in habit strength and in average exercise time. The group did not significantly regress on changes in average exercise time directly. However, while changes in habit strength significantly regressed on changes in average exercise time from baseline to the second survey, it did not significantly regress on it from the second to the third survey. Thus, while path coefficients for the study phase from baseline to the second survey supported Hypothesis 3, path coefficients for the phase from the second to the third survey did not support Hypothesis 3 due to non-significant regression from habit strength to average exercise time.

Electronic Supplementary Material 9 represents the total, direct, and indirect effects of changes in average exercise time for the path analysis of the sequential mediation model. During both phases, the total and indirect effects of the group and the total and direct effects of change in selfregulation were significant, and the direct effect of the group were not statistically significant. However, while the total 


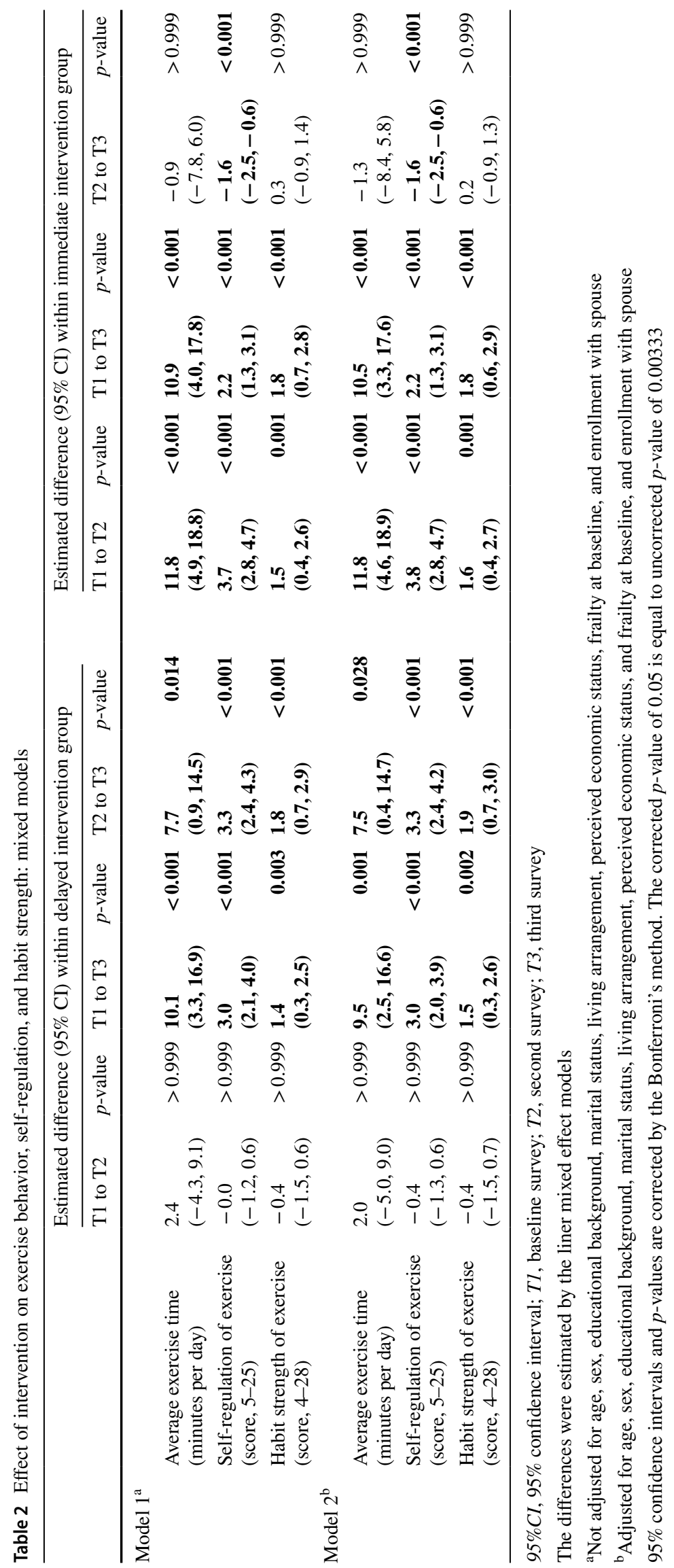



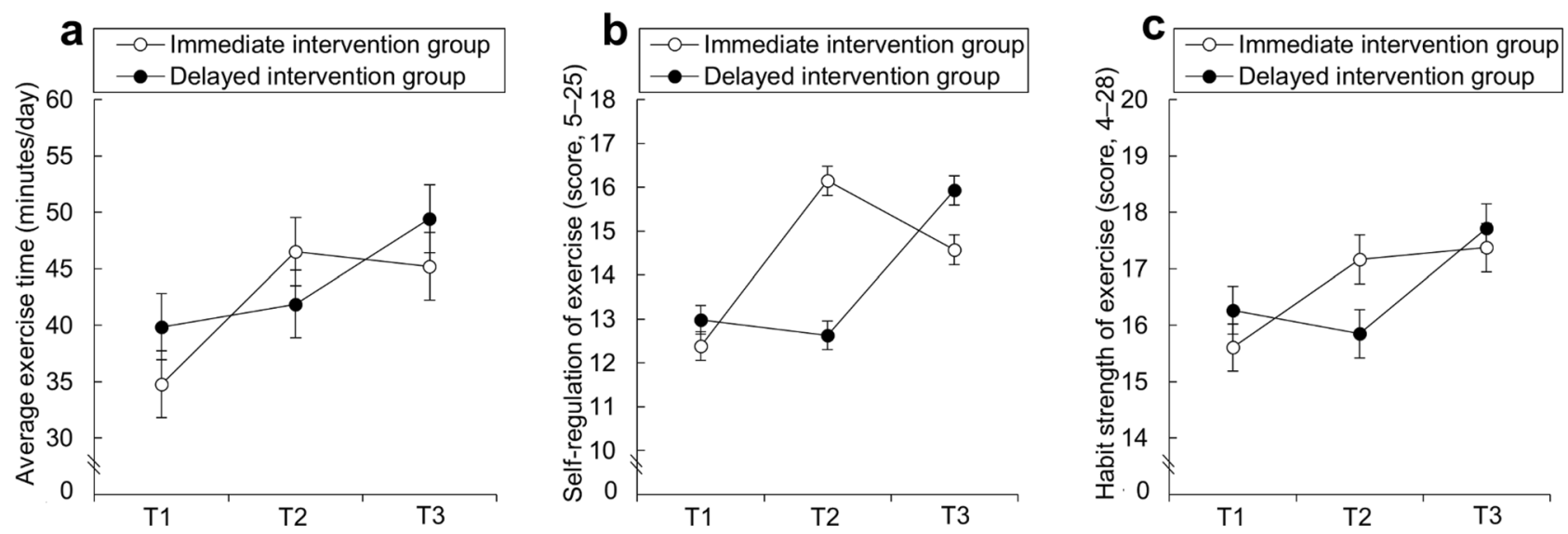

Fig. 2 Effect of intervention on a exercise behavior $\mathbf{b}$ self-regulation, and $\mathbf{c}$ and habit strength. Note.T1: baseline survey; T2: second survey; T3: third survey. Figures show estimated means of exercise behavior a, self-regulation $\mathbf{b}$, and habit strength $\mathbf{c}$ at each survey point. The error bars represent standard errors. Means and standard errors were esti-

and direct effects of changes in habit strength were significant during the study phase from baseline to the second survey, the total and direct effects of changes in habit strength were not significant during the phase from the second to the third survey. Therefore, corresponding to findings from the path coefficients, the total, direct, and indirect effects during the study phase from the second to the third survey did not support Hypothesis 3 due to non-significant total direct effects of habit strength, though these effects during the phase from baseline to the second survey supported it.

Another path model for the parallel mediation process is shown in Electronic Supplementary Material 10. The total, direct, and indirect effects for the parallel mediation model is displayed in Supplementary Material 11. The model fit indices of the parallel mediation model were poorer (model for baseline to the second survey, $\chi^{2}(4)=36.1(p<0.001)$, $\mathrm{CFI}=0.787$, TLI $=0.467$, RMSEA $=0.156$; model for the second to third survey, $\chi^{2}(6)=21.0(p=0.002), \mathrm{CFI}=0.855$, $\mathrm{TLI}=0.638$, RMSEA $=0.088)$ than the sequential mediation model (model for baseline to the second survey, $\chi^{2}(3)=7.7$ $(p=0.052), \mathrm{CFI}=0.969, \mathrm{TLI}=0.895, \mathrm{RMSEA}=0.069$; and the model for the second to third survey, $\chi^{2}(5)=3.3$ $(p=0.657)$, CFI $>0999$, TLI $>0.999$, RMSEA $<0.001)$. Thus, results support that the sequential mediation model was appropriate to fit the data.

\section{Discussion}

The present study found that exercise behavior was promoted after intervention in both immediate and delayed intervention groups, and that health literacy, habit strength, and socio-demographic factors did not moderate the intervention mated by the mixed effect models after adjustment of age, sex, educational background, marital status, living arrangement, perceived economic status, frailty at baseline, and enrollment with spouse

effect on exercise behavior in both groups. These findings show that self-regulation interventions can facilitate exercise behavior among older Japanese adults, regardless of their health literacy level, habit strength, and socio-demographic characteristics. Although recent systematic reviews and meta-analyses have supported the positive effects of selfregulation interventions on physical activity [18-21], there is little evidence pertaining to the older Japanese population. The present study contributes to confirming the role of self-regulation in physical activity promotion among older adults living in various regions. While people tend to decline in health literacy $[24,25]$ as they get older, decline in health literacy would not be major causes of the heterogeneous effects of self-regulation among older adults. Regardless of their health literacy levels, older adults could accept and incorporate self-regulation strategies into their daily exercise behaviors. Similar to the present study, previous studies on younger populations have shown that physical activity interventions are effective even for participants with low health literacy [30, 31]. Regarding the moderating role of habit strength, while previous studies of smoking behavior [35] and physical activity [36] among students confirmed its effects, a previous study for physical activity among adults in general [37] did not. Our research on older adults supports the previous findings drawn from studies of adults in general [37]. Although more research would be necessary to confirm the moderating role of habit strength, the present study, and findings related to adults in general [37], established habits might not seriously interfere with the development of new habits. In terms of practical settings, adequate acceptability of physical activity interventions among those with lower health literacy and higher habit strength would be favorable. Instead of health literacy and habit strength, 


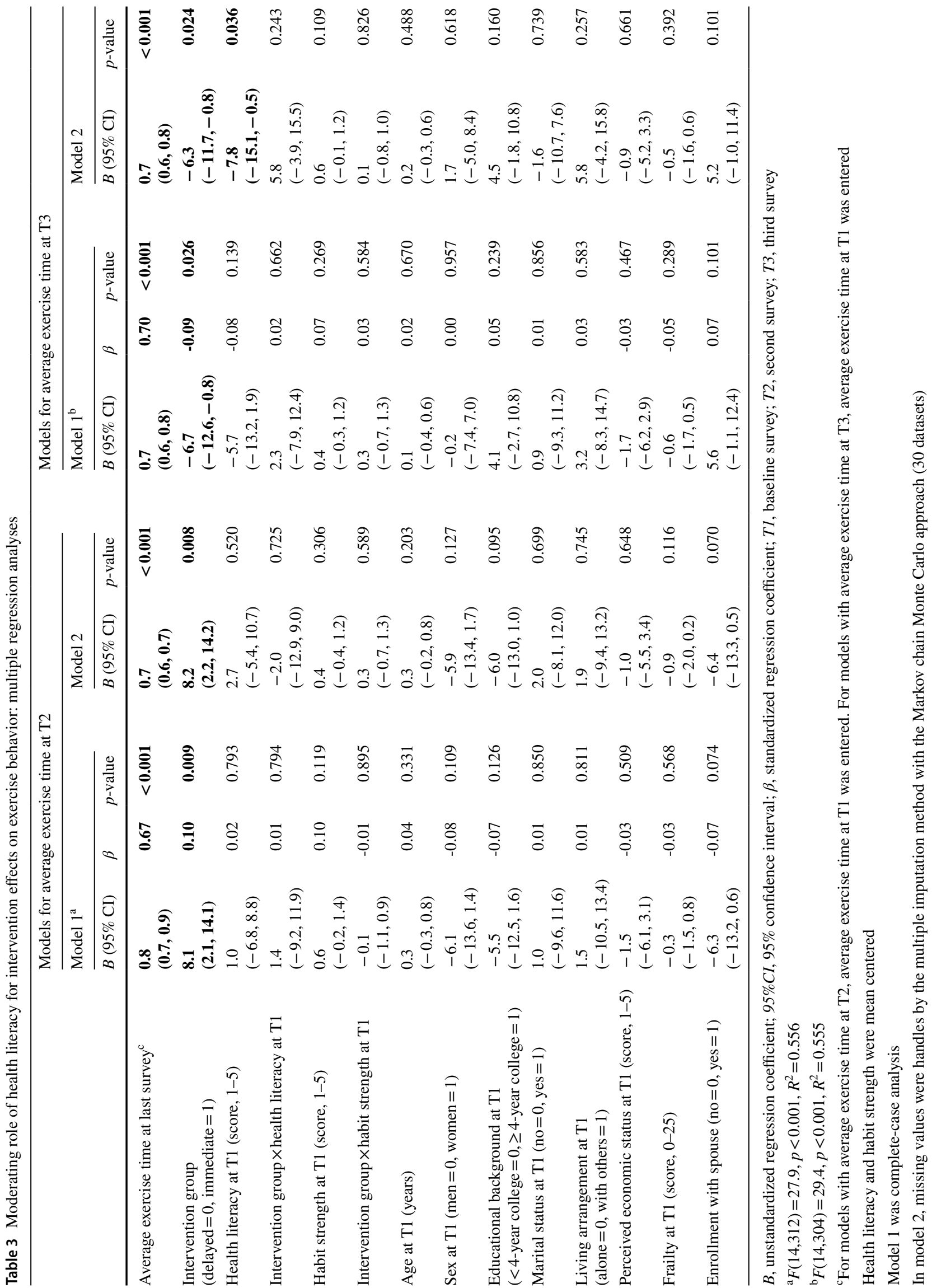




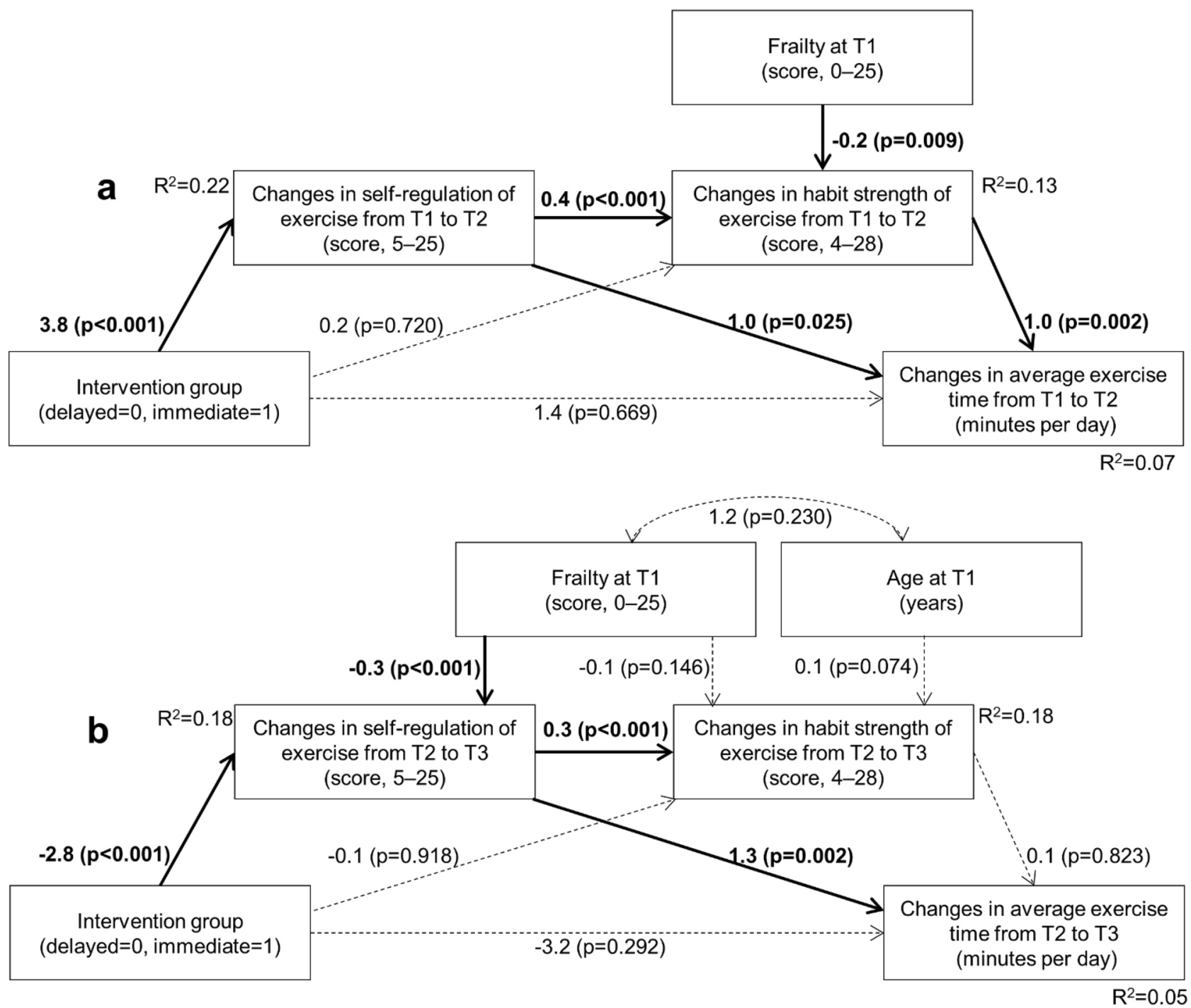

Fig. 3 Path models for sequential mediation process of intervention effects on exercise behavior. Note. T1: baseline survey; T2: second survey; T3: third survey. a represents the effects from the baseline to the second survey $\mathbf{a}$, and $\mathbf{b}$ represents the effects from the second to third surveys. The bold and dashed lines represent statistically significant and non-significant paths, respectively. Each change score

executive function might cause the heterogeneous effects of the self-regulation among older adult. Executive function represents higher-order cognitive abilities to control thought and action [64], and declines with aging [65]. Since self-regulation refers to one's behavior management skills, successful self-regulation of physical activity requires sufficient executive function [66]. Hall et al. [67] showed that the effects of implementation intention, a concept similar to self-regulation, are more relevant among older adults with higher executive function than among those with lower executive function. It is possible that most of the participants in the present study had adequate executive function. More represents the residualized change score. The model-fit indices were $\chi^{2}(3)=7.7(p=.052), \mathrm{CFI}=0.969, \mathrm{TLI}=0.895$, and $\mathrm{RMSEA}=0.069$ in the model for changes from the baseline to the second survey a, and $\chi^{2}(5)=3.3(p=0.657)$, CFI $>0.999$, TLI $>0.999$, and RMSEA $<0.001$ in the model for changes from the second to third survey b, respectively

extensive studies examining moderators, especially focusing on executive function, would be beneficial to confirm the effectiveness of self-regulation interventions among older adults.

For the mediating process, the present study found that the intervention indirectly influenced habit strength and exercise behavior, mediated by self-regulation. However, the present study did not show a clear relationship between habit strength and exercise behavior; a significant relationship was observed at the initial study phase (from baseline to the second survey), but not at the next study phase (from the second to the third survey). The mediating role 
of self-regulation on the relationship between intervention and exercise behavior confirms the successful manipulation of self-regulation by implementing the intervention in the present study. Furthermore, the findings about the mediating roles of self-regulation in the relationship between intervention and habit strength indicate that the employment of self-regulation would be an effective strategy for habit formation. This finding empirically supports the framework for understanding habit formation and its determinants [33, 40] and strengthens the current evidence about the strategies of habit [41]. However, the desirable effect of forming habit on behavior change was not replicable in the present study. One potential reason for the inconsistent results of habit-strengthto-behavior relationships might be that habit strength has multiple components: instigation habit and execution habit [68]. While instigation habits reflect the habit when deciding to perform certain behaviors, execution habits reflect the habit when actually beginning to perform certain behaviors [68]. Phillips and Gardner [69] reported that instigation habits significantly predicted exercise behavior, but that execution habits did not. The lack of careful discrimination of these two types of habit strength in the present study might have caused their contamination when answering the survey, leading to inconsistent results on the relationship between habit strength and exercise behavior. As a systematic review [70] stated that the relationship between habit strength and physical activity is still inconclusive due to the limited availability of evidence, and further detailed examinations are necessary to reveal the relationship between them.

As for the generalizability of the findings, participants in the present study are not representative of the older Japanese population. There were more women than men in the sample. While the study intervention was carried out in a typical Japanese urban environment, it is obvious that environmental resources, which are important determinants of leisure-time physical activity among older adults [71], are considerably different between urban and rural areas. The present study recruited participants via flyers inserted into the newspaper. The flyers stated that the study did not provide financial incentives for participation. Therefore, it is possible that those with low motivation would simply ignore the flyers or not be interested in the participation to the study. Most participants are likely to have been highly motivated. This would lead to sampling bias. Intention strength might not have been an important confounder and moderator of the intervention for this study, unlike other intervention-based studies (e.g., Pfeffer and Strobach [72]). The Health Action Process Approach [11] proposes that promoting planning (one strategy of self-regulation) is important when intentions are formed. Thus, the findings might not be generalizable to individuals with lower intention levels.

For the feasibility and translatability of the intervention to general practices, all intervention materials used in the present study were print-based and sent to all participants simultaneously via postal mail. No special knowledge, careful management, or larger resources are necessary for providing the materials. As shown in Supplementary Table 4, perceived adherence and acceptance of the intervention among the participants might be adequate. The intervention effects were not heterogeneous with regard to the participants' socio-demographic characteristics. Thus, the intervention of the present study would be feasible and translatable to general practice. Furthermore, a meta-analysis reported that interventions using electronic technology can increase $7.4 \mathrm{~min}$ of daily physical activity time among older adults [73]. As shown in Table 2, the present study increased $11.8 \mathrm{~min}$ and $7.6 \mathrm{~min}$ of daily exercise time in the immediate and delayed intervention groups, respectively. Thus, the clinical impact of the print-delivered intervention in the present study is equivalent to that of interventions using electronic technology. As $27.6 \%$ of older adults are still non-users of the Internet in Japan [74], interventions using electronic technology cannot approach a considerable proportion of older adults. The print-based interventions could compensate for this disadvantage of interventions using electronic technology.

The strength of the present study was the use of a crossover design. By employing this approach, the present study can confirm the replicability of the findings. However, this study has several limitations. First, as indicated above, most of the participants were highly motivated to engage in exercise behavior. Second, long-term maintenance of exercise behavior after the intervention was not followed. Third, the total physical activity was not assessed. Promoting exercise behavior is just one way to increase the total volume of physical activity. Measuring total physical activity, especially employing objective methods, would strengthen the scientific and practical values of the present study. Fourth, the validity and reliability of the measure for exercise behavior were not established. Especially, the present study did not restrict exercise behavior to moderate-to-vigorous intensity levels in accordance with current recommendations in Japan [2]. This is not consistent with global research trends on physical activity and health and the recommendations of the World Health Organization [1]. Fifth, the translation of the measure of habit strength did not align with rigorous procedures of scale developments (e.g., back translation). Sixth, the rationales of the intervention material were not rigorously constructed. The material was not based on specific health behavior theories/models. This intervention did not cover all major techniques to improve self-regulation and habit strength (e.g., feedback on behavior). Lack of such strategies may have weakened the intervention effects. Additionally, the first and second components of the intervention included techniques not typical of self-regulation strategies in health behavior [13] such as "information about 
health consequences" and "social support." The inclusion of such techniques may contaminate the results. Examining other potential mediators (e.g., beliefs about consequences) would have been also beneficial [38]. Finally, adherence to the intervention was measured only by self-report. Nonetheless, the present study contributes to a better understanding of the effects of self-regulation interventions on exercise behavior among older adults.

In conclusion, although the mediating roles of habit strength for the intervention effects are still inconclusive, the findings of the present study show that self-regulation intervention can promote exercise behavior among older adults, regardless of their health literacy level, habit strength, and socio-demographic characteristics.

Supplementary Information The online version contains supplementary material available at https://doi.org/10.1007/s12529-021-10049-3.

Acknowledgements I wish to thank Ms. Mariko Yamashita, a staff member of the health and healthcare division in the Nada ward office, for her helpful support in the recruitment of the participants and the offer of the intervention materials.

Funding This work was supported by the University's Urban Innovation Kobe (FY2020, A20109), Kobe City Government.

\section{Declarations}

Ethical Approval All procedures performed in studies involving human participants were in accordance with the ethical standards of the institutional and/or national research committee and with the 1964 Helsinki declaration and its later amendments or comparable ethical standards.

Informed Consent Informed consent was obtained from all individual participants included in the study.

Conflict of Interest The author declares no competing interests.

Disclaimer Kobe City Government had no role in the study design, data collection and analysis, decision to publish, or preparation of the manuscript.

\section{References}

1. World Health Organization. WHO Guidelines on physical activity and sedentary behavior. https://apps.who.int/iris/rest/bitstreams/ 1315866/retrieve. Accessibility verified October 21, 2021.

2. Ministry of Health, Labour, and Welfare. Kenko dukuri no tameno shintai-katsudo kijun 2013 [Recommended levels of physical activity for promoting health 2013]. (in Japanese) https://www. mhlw.go.jp/stf/houdou/2r9852000002xple-att/2r9852000002xpqt. pdf Accessibility verified October 21, 2021.

3. Inoue S, Ohya Y, Tudor-Locke C, Tanaka S, Yoshiike N, Shimomitsu T. Time trends for step-determined physical activity among Japanese adults. Med Sci Sports Exerc. 2011;43(10):1913-9.

4. Barnett I, van Sluijs E, Ogilvie D, Wareham NJ. Changes in household, transport and recreational physical activity and television viewing time across the transition to retirement: longitudinal evidence from the EPIC-Norfolk cohort. J Epidemiol Community Health. 2014;68(8):747-53.

5. Mann T, de Ridder D, Fujita K. Self-regulation of health behavior: social psychological approaches to goal setting and goal striving. Heal Psychol. 2013;32(5):487-98.

6. de Ridder D, Wit J. Self-regulation in health behavior: concepts, theories, and central issues. In: de Ridder D, Wit J, editors. Selfregulation in Health Behavior. Chichester, UK: John Wiley \& Sons Ltd; 2006. p. 1-23.

7. Kwasnicka D, Dombrowski SU, White M, Sniehotta F. Theoretical explanations for maintenance of behaviour change: a systematic review of behaviour theories. Health Psychol Rev. 2016;10(3):277-96.

8. Hall PA, Fong GT. Temporal self-regulation theory: a model for individual health behavior. Health Psychol Rev. 2007;1(1):6-52.

9. Bandura A. Self-efficacy: The exercise of control. New York, NY: Freeman; 1997.

10. Prochaska JO, DiClemente CC. Stages and processes of selfchange of smoking: Toward an integrative model of change. $\mathrm{J}$ Consult Clin Psychol. 1983;51(3):390-5.

11. Schwarzer R, Schuz B, Ziegelmann JP, Lippke S, Luszczynska A, Scholz U. Adoption and maintenance of four health behaviors: theory-guided longitudinal studies on dental flossing, seat belt use, dietary behavior, and physical activity. Ann Behav Med. 2007;33(2):156-66.

12. Hennessy EA, Johnson BT, Acabchuk RL, McCloskey K, Stewart-James J. Self-regulation mechanisms in health behavior change: a systematic meta-review of meta-analyses, 2006-2017. Health Psychol Rev. 2020;14(1):6-42.

13. Spring B, Champion KE, Acabchuk R, Hennessy EA. Self-regulatory behaviour change techniques in interventions to promote healthy eating, physical activity, or weight loss: a meta-review. Health Psychol Rev. in press. https://doi.org/10.1080/17437199.2020.1721310.

14. Baruth M, Wilcox S, Dunn AL, et al. Psychosocial mediators of physical activity and fitness changes in the activity counseling trial. Ann Behav Med. 2010;39(3):274-89.

15. Anderson ES, Wojcik JR, Winett RA, Williams DM. Socialcognitive determinants of physical activity: the influence of social support, self-efficacy, outcome expectations, and selfregulation among participants in a church-based health promotion study. Health Psychol. 2006;25(4):510-20.

16. Murray JM, Brennan SF, French DP, Patterson CC, Kee F, Hunter RF. Mediators of behavior change maintenance in physical activity interventions for young and middle-aged adults: a systematic review. Ann Behav Med. 2018;52(6):513-29.

17. French DP, Olander EK, Chisholm A, Mc SJ. Which behaviour change techniques are most effective at increasing older adults' self-efficacy and physical activity behaviour? A systematic review Ann Behav Med. 2014;48(2):225-34.

18. Kanejima Y, Kitamura M, Izawa KP. Self-monitoring to increase physical activity in patients with cardiovascular disease: a systematic review and meta-analysis. Aging Clin Exp Res. 2019;31(2):163-73.

19. Duan Y, Shang B, Liang W, Du G, Yang M, Rhodes RE. Effects of eHealth-based multiple health behavior change interventions on physical activity, healthy diet, and weight in people with noncommunicable diseases: Systematic review and meta-analysis. J Med Internet Res. 2021;23(2):e23786.

20. Muellmann S, Forberger S, Möllers T, Bröring E, Zeeb H, Pischke CR. Effectiveness of eHealth interventions for the promotion of physical activity in older adults: a systematic review. Prev Med (Baltim). 2018;108:93-110.

21. Stockwell S, Schofield P, Fisher A, et al. Digital behavior change interventions to promote physical activity and/or reduce sedentary behavior in older adults: a systematic review and meta-analysis. Exp Gerontol. 2019;120:68-87. 
22. Rhodes RE, Janssen I, Bredin SSD, Warburton DER, Bauman A. Physical activity: health impact, prevalence, correlates and interventions. Psychol Health. 2017;32(8):942-75.

23. Conner M, Norman P. Health behaviour: current issues and challenges. Psychol Health. 2017;32(8):895-906.

24. Kobayashi LC, Wardle J, Wolf MS, von Wagner C. Cognitive function and health literacy decline in a cohort of aging English adults. J Gen Intern Med. 2015;30(7):958-64.

25. Paasche-Orlow MK, Parker RM, Gazmararian JA, Nielsen-Bohlman LT, Rudd RR. The prevalence of limited health literacy. J Gen Intern Med. 2005;20(2):175-84.

26. Buja A, Rabensteiner A, Sperotto M, et al. Health literacy and physical activity: a systematic review. J Phys Act Health. 2020;17(12):1259-74.

27. Paasche-Orlow MK, Wolf MS. The causal pathways linking health literacy to health outcomes. Am J Health Behav. 2007;31(Suppl 1):S19-26.

28. Osborn CY, Paasche-Orlow MK, Bailey SC, Wolf MS. The mechanisms linking health literacy to behavior and health status. Am J Health Behav. 2011;35(1):118-28.

29. Sørensen K, Van den Broucke S, Fullam J, et al. Health literacy and public health: a systematic review and integration of definitions and models. BMC Public Health. 2012;12(1):80.

30. Dominick GM, Dunsiger SI, Pekmezi DW, et al. Moderating effects of health literacy on change in physical activity among Latinas in a randomized trial. J Racial Ethn Heal Disparities. 2015;2(3):351-7.

31. Hartman SJ, Dunsiger SI, Bock BC, et al. Physical activity maintenance among Spanish-speaking Latinas in a randomized controlled trial of an Internet-based intervention. J Behav Med. 2017;40(3):392-402.

32. Hagger MS. Habit and physical activity: theoretical advances, practical implications, and agenda for future research. Psychol Sport Exerc. 2019;42:118-29.

33. Lally P, Gardner B. Promoting habit formation. Health Psychol Rev. 2013;7(sup1):S137-58.

34. Hofmann W, Friese M, Wiers RW. Impulsive versus reflective influences on health behavior: a theoretical framework and empirical review. Health Psychol Rev. 2008;2(2):111-37.

35. Webb TL, Sheeran P, Luszczynska A. Planning to break unwanted habits: habit strength moderates implementation intention effects on behaviour change. Br J Soc Psychol. 2009;48(3):507-23.

36. Maher JP, Conroy DE. Habit strength moderates the effects of daily action planning prompts on physical activity but not sedentary behavior. J Sport Exerc Psychol. 2015;37(1):97-107.

37. Di Maio S, Keller J, Hohl DH, Schwarzer R, Knoll N. Habits and self-efficacy moderate the effects of intentions and planning on physical activity. Br J Health Psychol. 2021;26(1):50-66.

38. Rhodes RE, Boudreau P, Josefsson KW, Ivarsson A. Mediators of physical activity behaviour change interventions among adults: a systematic review and meta-analysis. Health Psychol Rev. 2021;15(2):272-86.

39. Gardner B, Lally P. Modelling habit formation and its determinants. In: Verplanken B, editor. The psychology of habit. Cham, Switzerland: Springer; 2018. p. 207-29.

40. Gardner B. A review and analysis of the use of 'habit' in understanding, predicting and influencing health-related behaviour. Health Psychol Rev. 2015;9(3):277-95.

41. Fritz H, Hu Y-L, Gahman K, Almacen C, Ottolini J. Intervention to modify habits: a scoping review. OTJR Occup Particip Heal. 2020;40(2):99-112.

42. Dwan K, Li T, Altman DG, Elbourne D. CONSORT 2010 statement: extension to randomised crossover trials. BMJ. 2019;366:14378.

43. Faul F, Erdfelder E, Buchner A, Lang AG. Statistical power analyses using $\mathrm{G}^{*}$ Power 3.1: tests for correlation and regression analyses. Behav Res Methods. 2009;41(4):1149-1160.
44. Yamada M, Nishiguchi S, Fukutani N, Aoyama T, Arai H. Mailbased intervention for sarcopenia prevention increased anabolic hormone and skeletal muscle mass in community-dwelling Japanese older adults: The INE (Intervention by Nutrition and Exercise) Study. J Am Med Dir Assoc. 2015;16(8):654-60.

45. Uemura K, Yamada M, Okamoto H. Effects of active learning on health literacy and behavior in older adults: a randomized controlled trial. J Am Geriatr Soc. 2018;66(9):1721-9.

46. Seino S, Nishi M, Murayama $\mathrm{H}$, et al. Effects of a multifactorial intervention comprising resistance exercise, nutritional and psychosocial programs on frailty and functional health in communitydwelling older adults: a randomized, controlled, cross-over trial. Geriatr Gerontol Int. 2017;17(11):2034-45.

47. Shimada H, Makizako H, Doi T, et al. Effects of combined physical and cognitive exercises on cognition and mobility in patients with mild cognitive impairment: a randomized clinical trial. J Am Med Dir Assoc. 2018;19(7):584-591.

48. Michie S, Richardson M, Johnston M, et al. The behavior change technique taxonomy (v1) of 93 hierarchically clustered techniques: building an international consensus for the reporting of behavior change interventions. Ann Behav Med. 2013;46(1):81-95.

49. Murakami K, Hashimoto H, Lee JS, Kawakubo K, Mori K, Akabayashi A. Distinct impact of education and income on habitual exercise: a cross-sectional analysis in a rural city in Japan. Soc Sci Med. 2011;73(12):1683-8.

50. Chen T, Lee JS, Kawakubo K, et al. Features of perceived neighborhood environment associated with daily walking time or habitual exercise: differences across gender, age, and employment status in a community-dwelling population of Japan. Environ Health Prev Med. 2013;18(5):368-76.

51. Sugisawa H, Harada K, Sugihara $Y$, Yanagisawa S, Shimmei M. Time perspectives as mediators of the associations between socioeconomic status and health behaviours in older Japanese adults. Psychol Health. 2020;35(8):1000-16.

52. Ministry of Health, Labour, and Welfare. Kokumin kenko eiyo chosa [Japan National Health and Nutrition Survey] (in Japanese). https://www.mhlw.go.jp/bunya/kenkou/kenkou_eiyou_ chousa.html. Accessibility verified October 21, 2021.

53. Takeda N, Oka K, Sakai K, Nakamura Y. Seijin ni okeru undo ni kansuru koudouteki sukiru to undo kodo no hen-yo suteji no kanren [The relationship between exercise behavioral skills and the stages of change for exercise behavior among Japanese adults]. [Kodo Igaku Kenkyu] Japanese J Behav Med. 2009;14(1):8-14. (in Japanese)

54. Gardner B, Abraham C, Lally P, de Bruijn G-J. Towards parsimony in habit measurement: testing the convergent and predictive validity of an automaticity subscale of the Self-Report Habit Index. Int J Behav Nutr Phys Act. 2012;9:102.

55. Verplanken B, Orbell S. Reflections on past behavior: a self-report index of habit strength. J Appl Soc Psychol. 2003;33(6):1313-30.

56. Rebar AL, Gardner B, Rhodes RE, Verplanken B. The measurement of habit. In: Verplanken B, editor. The psychology of habit. Cham, Switzerland: Springer; 2018. p. 31-49.

57. Ishikawa H, Nomura K, Sato M, Yano E. Developing a measure of communicative and critical health literacy: a pilot study of Japanese office workers. Health Promot Int. 2008;23(3):269-74.

58. Arai H, Satake S. English translation of the Kihon Checklist. Geriatr Gerontol Int. 2015;15(4):518-9.

59. Ministry of Health, Labour and Welfare of Japan. Kaigo-yobo no tame no Seikatsu-kino-hyokani kansuru manyuaru: kaiteiban [Revised manual for life function assessment for prevention of long-term care]. https://www.mhlw.go.jp/topics/2009/05/dl/ tp0501-1c.pdf (in Japanese) Accessibility verified June 25, 2021.

60. Satake S, Senda K, Hong Y-J, et al. Validity of the Kihon Checklist for assessing frailty status. Geriatr Gerontol Int. 2016;16(6):709-15. 
61. Hu L, Bentler PM. Cutoff criteria for fit indexes in covariance structure analysis: conventional criteria versus new alternatives. Struct Equ Model A Multidiscip J. 1999;6(1):1-55.

62. Cavallo DN, Brown JD, Tate DF, DeVellis RF, Zimmer C, Ammerman AS. The role of companionship, esteem, and informational support in explaining physical activity among young women in an online social network intervention. J Behav Med. 2014;37(5):955-66.

63. Gunnell KE, Crocker PRE, Mack DE, Wilson PM, Zumbo BD. Goal contents, motivation, psychological need satisfaction, wellbeing and physical activity: a test of self-determination theory over 6 months. Psychol Sport Exerc. 2014;15(1):19-29.

64. Miller EK, Wallis JD. (2009). Executive function and higherorder cognition: definition and neural substrates. In: Squire LR, ed. Encyclopedia of neuroscience. Oxford: Academic Press. 2009: 99-104.

65. Lessov-Schlaggar CN, Swan GE, Reed T, Wolf PA, Carmelli D. Longitudinal genetic analysis of executive function in elderly men. Neurobiol Aging. 2007;28(11):1759-68.

66. Buckley J, Cohen JD, Kramer AF, McAuley E, Mullen SP. Cognitive control in the self-regulation of physical activity and sedentary behavior. Front Hum Neurosci. 2014;8(September):747.

67. Hall PA, Zehr C, Paulitzki J, Rhodes R. Implementation intentions for physical activity behavior in older adult women: an examination of executive function as a moderator of treatment effects. Ann Behav Med. 2014;48(1):130-6.

68. Gardner B, Phillips LA, Judah G. Habitual instigation and habitual execution: definition, measurement, and effects on behaviour frequency. Br J Health Psychol. 2016;21(3):613-30.
69. Phillips LA, Gardner B. Habitual exercise instigation (vs. execution) predicts healthy adults' exercise frequency. Health Psychol. 2016;35(1):69-77.

70. Feil K, Allion S, Weyland S, Jekauc D. A systematic review examining the relationship between habit and physical activity behavior in longitudinal studies. Front Psychol. 2021;12(March):626750.

71. Van Cauwenberg J, Nathan A, Barnett A, Barnett DW, Cerin E. Relationships between neighbourhood physical environmental attributes and older adults' leisure-time physical activity: a systematic review and meta-analysis. Sport Med. 2018;48(7):1635-60.

72. Pfeffer I, Strobach T. Influence of a planning intervention on physical activity behavior: the moderating role of intentions and executive functions in a randomized controlled trial. Int J Behav Med. 2020;27(5):506-19.

73. Núñez de Arenas-Arroyo S, Cavero-Redondo I, Alvarez-Bueno C, Sequí-Domínguez I, Reina-Gutiérrez S, Martínez-Vizcaíno V. Effect of eHealth to increase physical activity in healthy adults over 55 years: a systematic review and meta-analysis. Scand J Med Sci Sports. 2021;31(4):776-789.

74. Ministry of Internal affairs and commutation of Japan. Reiwagannendo tsushin riyo doko chosa: setai kosei-in hen [Communications usage trend survey 2019: Results for household members]. https://www.e-stat.go.jp/stat-search/file-download?statInfId= 000031952129\&fileKind=1 (in Japanese) Accessibility verified June 25, 2021.

Publisher's Note Springer Nature remains neutral with regard to jurisdictional claims in published maps and institutional affiliations. 